\section{CLINICAL LECTURES ON}

\author{
CERTAIN DISEASES OF THE CHEST.
}

DELIVERED AT THE

LIVERPOOL NORTHERN HOSPITAL.

\author{
BY A. T. H. WA TERS, M.D., F.R.C.P., \\ Physician to the Hospital.
}

LECTURE IV.-PNeumonia (continued).

Gentlemen,--In my last lecture, I pointed out to you the modes of treatment which I think are most to be depended on in cases of pneumonia; and I now wish to draw your attention to the details of some cases in which the principles I have referred to have been practically applied.

There is one circumstance to which I have not specially alluded, but which is of much importance in reference to the treatment of this disease; viz., the constitutional condition of the patients who are attacked by it. There can be no doubt that in many patients there has been some deterioration of health which has acted as the predisposing cause of the disease ; but, at the same time, it is quite certain that cases occasionally occur in which there has been no previous ill-health. In such patients, exposure to wet or to cold, or to both, has been the exciting cause of the attack. Instances of this kind we sometimes meet with in our wards, in strong, active labourers or seamen, who are suddenly struck down by the disease. At the same time, I believe that in private practice you will very rarely meet with such cases. Most of those attacked with pneumonia, whom you will be called upon to treat in private practice, and especially the more wealthy of your patients, will be people not exposed to great hardships, nor to the inclemencies of the weather; and in these persons you will generally be able to trace some antecedent depressing circumstances, some deterioration of health, or some organic disease which has predisposed to the pneumonic attack. I need not now impress on you the importance of inquiring into all these points when you come to consider the question of treatment, as I shall have to refer to them more particularly hereafter. Let me, however, here remark that in all.cases, not only of pneumonia, but of other acute diseases, you should ascertain at the earliest possible period, by a careful examination, whether your patient is the subject of any organic disease of the kidneys, heart, or liver.

If there be one symptom more important than another in pneumonia, and which affords a safer guide to treatment than any other single phenomenon which the disease presents, it is, I think, the pulse. Had we some means of correctly estimating the character of the pulse, we should have less difficulty in judging of the appropriate remedies for the various inflammatory affections we meet with. It is not simply the frequency of the pulse which is important, but the amount of arterial tension which it indicates, and its general character, as showing whether a stimulating or an opposite line of treatment is required. To estimate aright this latter point is one of the most difficult problems in medicine, and can only result - with our present modes of investigation-either from unusual powers of discrimination or prolonged clinical experience.

I dwell upon this subject, as I would also dwell on the importance of other symptoms in this disease, such. as the respiration, the hot skin, the expression of the countenance, the voice, the condition of the nervous system; for, after all, when the question of treatment comes to be talien into consideration, it is these altered physiological conditions which mainly guide us. Our physical diagnosis tells us how much of one lung, or of both lungs, is involved in the disease; but it does not point out the line of treatment to be adopted. One patient with a small portion of lung involved in pneumonia may present more severe constitutional symptoms than another who has double the amount of tissue inflamed; and, as it is the patient you wish to cure, so your remedies must be addressed to the relief of the constitutional symptoms which are present. As a rule, it may perhaps be said that in pneumonia, when the pulse is below 1๑0, the case is not a grave one, and will yield to treatment of a simple character; but when the pulse rises to 110,120 , or upwards, the case assumes a much more serious aspect. The more frequent the pulse, as a ralle, the greater is the need for stimulants; and it is very remarkable to see the effect they produce on the pulse when it is abnormally frequent in some cases of pneumonia. But it is not every quiok pulse, not every pulse of 120 , which is benefited by alcoholic stimulants, as the following cases will show.

Leopold A., 29 years of age, a Norwegian sailor, moderately stout, was admitted into the hospital on November 2oth, 1863 . On admis- sion, he complained of pain in the chest and cough, and the physical signs of bronchitis were present. He was ordered turpentine fomentations to the chest, and stimulating expectorants, with six ounces of port wine daily, and beef-tea. No improvement followed; and, on the 22nd, symptoms of pneumonia set in.

On the 23rd, the skin was hot; the pulse was 132 . There was marked crepitation at the base of the left lung, with dulness on percussion; the right lung being unaffected.

On the 24th, the pulse was 128, and strong. There was great anxiety of countenance, and much general distress; the skin was very hot, and the sputum rust-coloured. Crepitation and dulness were found over the lower half of the left lung, with some crepitation over the base of the right. Up'to this time, no change had been made in the treatment prescribed, and the patient was evidently getting worse. The stimulating expectorants and the wine were stopped; and a fourth of a grain of tartarised antimony, with acetate of ammonia, was ordered every three hours. In the evening, a small quantity of wine was given in mistake; but none was afterwards taken until convalescence was established.

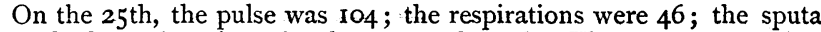
were frothy and copious, but less rust-coloured. There was no crepitation over the right lung, but slight dulness was found. There was marked crepitation all over the left lung, with deficient expansion of the left side of the chest.

On the 26 th, the pulse had fallen to 88 ; the respirations to 32 . The sputa were frothy and copious. The antimony was ordered to be taken during the day only.

On the $27^{\text {th }}$, the pulse was 80 ; the respirations were 32 .

On the 28 th, they were respectively the same. The breathing was natural over the right lung, but slight dulness remained at the back of the left. The antimony was stopped; and ipecacuanha wine, with tincture of squills, ordered; together with four ounces of port wine and chop diet.

On the 29 th, the pulse was 76 ; the respirations were 28 .

On December Ist, the pulse was 90 ; the respirations were 24 . There was some return of crepitation on the left side in front, with slight fever. The wine was omitted; it appeared to act prejudicially.

From this date, the case progressed satisfactorily. On the 8 th, the pulse and respirations were normal; and on the $\mathrm{I} I$ th the patient was discharged well.

Here was a frequent pulse ; and yet stimulants only served to increase the urgency of the symptoms, which, however, rapidly yielded to the administration of antimony. Now let me refer you to another case.

D. D. M., a Frenchman, was admitted into the hospital on January 23rd, 1865. I have already alluded to the case in speaking of the early physical signs of pneumonia. The patient was a fireman on board a steamer just arrived from Rotterdam. The weather had been very cold, and the man had been exposed to great variations of temperature. He was perfectly well, he said, two days before he came to the hospital; but he had since felt pains about his chest, and had suffered from difficulty of breathing. He was of moderate height, of somewhat spare build, and fifty-five years of age. I saw him shortly after he was admitted. I found him with a hot and dry skin, flushed face, a furred tongue, a pulse of 100 , full and strong, quick respiration, and occasional cough. On examining his chest, we found the movement and resonance good everywhere; and the breath-sounds normal, except over the lower and back part of the left lung, where there was a loud, harsh, respiratory murmur. No crepitation could be heard anywhere. I expressed an opinion that this was a case of pneumonia in the early stage, and that before long the harsh respiration heard over the left lumg would be replaced by crepitation. I thought this would be a good opportunity for trying the effects of alcoholic stimulants in the onset of the disease. Nothing had been done for the patient before his admission. I accordingly, rather in the way of experiment than otherwise-for I was doubtful of any good result being produced-ordered a tablespoonful of brandy to be given every three hours, and turpentine stupes to: be applied to the chest. This treatment was commenced about one o'clock P.M. I left directions with the house-surgeon that the brandy:should not be discontinued, unless it appeared to do harm. 'The following are the notes of the progress of the case.

9 P.M. 'He appeared much wosse; had great dyspnca. Pulse 120, very full and strong, incompressible; respiration 40. We complained of a sense of oppression in the chest, and a ifeeling of suffocation; eheeks very flushed; great thirst. Crepitant honchus weard at the left base. He was ordered to continue the brandy, and to have san ounce of the following mixture every fourth hour.

$\mathrm{B}$ Ammoniæ carbon. $\mathrm{B} \mathrm{ij}$; spirit. chloroformi $3 \mathrm{iij}$; misturæe camphoræ दviij. M.

II P. M. He had had one dose of the mixture, and seemed still worse than at nine o'clock. The dyspnœa was so urgent that he could not lie 
down, and was obliged to be propped up in a bed-chair. Finding that the stimulating treatment was to all appearances doing the patient harm, the house-surgeon very wisely stopped it, and ordered the following mixture to be given-an ounce every three hours.

Bo Liquor. ammon. acetat. (Brit. Ph.) $3 \mathrm{ij}$; vini ipecac. $3 \mathrm{ij}$; vini antim. potassio-tart. $3 \mathrm{ij}$; spir. ether. sulph. comp. $3 \mathrm{j}$; aquæ q. s. ad そviij. $M$.

I wish you carefully to note the result of these measures.

On January 24 th, at 2 P.M., he was very much better. He said he began to improve after the second dose of the mixture. Pulse 92, less strong and full. Respiration 24. He had slight pain in the front of the chest, but the sensation of oppression was almost gone. The skin was less hot, and the face less flushed. The expectoration was frothy, not rust-coloured. There was diminished movement of the left side, and dulness of the lower part of the left lung, with abundant crepitant rates. He was ordered to continue the mixture and fomentations.

9 P.M. Pulse 100. Respiration 30. He had been rather sick this evening. He was ordered to take the mixture every four hours.

January 25th. Pulse 100. Respiration 28. Skin moist. The mix ture caused sickness. The expectoration was very tenacious and rustcoloured. The dulness at the left base was not so marked; crepitation abundant. He was ordered a tablespoonful of port wine every three hours, and to continue the mixture without the antimony.

I omitted the antimony on account of the sickness, and again tried the effects of stimulants in small quantities.

January 26th, 10 A.M. Pulse 100. Respiration 26. Tongue cleaner. He had no pain in the chest. The sickness had ceased. The expectoration was frothy and abundant. Crepitation was coarser than on the previous day. A blister was ordered to be applied to the left side, and an ounce of the following mixture every four hours.

Bo Vini ipecac. 3 iss; spirit. ether. sulph. comp. 3 iss; aquæ q. s. ad ऊvj. M.

January 27 th. He had passed a bad night on account of cough and dyspnœe. The expectoration was very tenacious and rust-coloured. There was tubular breathing at the left base; less crepitation. Rough and loud breathing was heard at the right base. He was ordered by the house-surgeon a tablespoonful of brandy every two hours.

2 P.M. Pulse 108. Distinct crepitation was heard at the right base. It was evident, from the symptoms which the patient presented when seen by me at two o'clock, that there was a return of the inflammation, and that the right lung was becoming involved. I felt convinced that the stimulating treatment was doing harm, and I accordingly stopped it al. together. I gave no more alcohol till convalescence was fairly established. $\mathrm{He}$ was ordered an ounce of the following mixture every two hours.

B Antim. potassio-tartrat. gr. j; tincturæ opii mxx; aquæ q. s. ad 弓vj. 9 P.M. He felt much better. The cough was diminished; and the expectoration more frothy. Pulse 104. Respiration 30. Abundant crepitation was heard on the right side.

January 28th. Pulse 96. Respiration 24. He had passed an excellent night. He breathed quite easily, and had much less cough. The physical signs were improving. He was ordered to take the mixture every four hours.

January 29th. Pulse 88. Respiration 24

January 30th. Pulse 88. Respiration 24. He was convalescent; and was ordered to take the mixture three times a day, and to have four ounces of port wine daily.

January 31st. Pulse 84. Respiration 24. He had been rather sick; otherwise he was going on well. The mixture was stopped, and he was ordered six ounces of wine daily, and a chop for dinner.

February Ist. Pulse 80. Respiration 20. The percussion-sound at the right base was almost natural. There were dulness and tubular breathing at the left base.

February 4th. Percussion and respiration were normal on the right side. He was ordered quinine, four ounces of port wine, and two ounces of brandy daily.

February 15 th. Respiration and percussion were normal on the left side.

February 22nd. He was discharged well.

I have referred at length to this case, because I think it is a very in. structive one; especially when compared with other cases which I shall refer to. It serves to show very forcibly that, as I said before, it is not every case of quick pulse which requires stimulants. When I tried the stimulants first, it was at the very onset of the disease, and there cannot be a doubt that they proved prejudicial; when I resorted to them the second time, the disease was subsiding, but two days of the treatment caused a relapse and a return of the unfavourable symptoms, which dis. appeared when the alcohol was omitted. But, although I have referred to these two cases with the view of showing you the injurious effects that alcoholic stimulants seemed to have on them, you must by no means conclude that there are no cases in which stimulants may not be advantageously given very early in the disease. Let me refer you to the following case, which is interesting in two particulars: $I$, as presenting a peculiar feature of the pulse; and 2, as showing the beneficial effects of a stimulating treatment.

Robert R., 23 years of age, a sailor, was admitted into the hospital on October 18th, 1866. He said he had always enjoyed good health, but was of somewhat intemperate habits. Three days before admission, after exposure to cold, he had shivering and sickness followed by pain in the right side.

On admission, the pulse was i12, and slightly intermittent. No marked abnormal sounds were heard on auscultation. He was ordered by the house-surgeon, a mustard poultice, to be followed by a linseedmeal poultice; and an ounce of the following mixture every four hours.

Bo Ammon. carb. gr. xxij; liquoris ammon. acetatis zij; spirit. etheris chlorici ३iss; aquæ puræ q. s. ad ऊrj. M.

October 19th, II A.M. He had passed a bad night. Pulse 120, intermittent; copious expectoration.

I did not see the patient till 3 P.M. of this day. The pulse had then fallen to 92, and was very intermittent. The expectoration was rustcoloured. There was a troublesome cough. There was bronchial breathing in the right axilla. The heart-sounds were normal. He was ordered to continue the ammonia, and to have a tablespoonful of brandy every hour and a half, witk: beef-tea, gruel, and milk.

On the 2oth, the pulse was still intermittent; crepitation was heard over the back of the right lung. The right cheek was much redder and hotter than the left.

October 2 Ist. He had passed a better night. Pulse 104, more regular. He had been purged several times. He was ordered to have five grains of Dover's powder immediately.

October 22nd. Pulse 84, very slightly intermittent.

October 23rd. Pulse 72, regular. There was slight dulness and bronchophony at the back of the right lung. Faint crepitation in the front.

I need not follow the daily details of the case. The patient steadily improved. He took the eight ounces of brandy daily till the 27 th, when the quantity was diminished to six ounces. On the 25 th, he was ordered quinine, which he took until discharged on Nov: 9th.

The points of interest to which I wish to draw your attention in the case are-the intermittent pulse, and the beneficial effects of the stimulants which were administered. The intermittent pulse is rarely met with in pneumonia, apart from organic disease of the heart. In this man there was no valvular affection of the heart; and there was no reason to suppose that the muscular substance was the seat of disease. The intermission of the pulse was simply a functional disturbance, the result of want of power, of deficient innervation. It afforded me a strong indi. cation for the line of treatment to be adopted. You will meet with this intermission of the pulse in various functional disorders-in stomach diseases, in functional disorders of the liver, etc. - but wherever you meet with it in acute inflammatory affections, I believe it is invariably an indication for the free use of stimulants. The quantity of stimulants given in this case, eight ounces-a third of a bottle - of brandy was not large; but continued as it was for eight days, after which only six ounces were given daily, it represents a considerable quantity of alcohol taken into the system. The results cannot be considered otherwise than satisfactory. The treatment was commenced early in the disease, and was directed to meet that condition of the patient, the most prominent fea. ture in which was the feeble and intermittent pulse.

There is another circumstance mentioned in the notes of the case which is of some interest-the increased temperature of one cheek, the right, the side of the pneumonia. This symptom is one I have frequently observed, and it has been made the subject of special observa. tion by an eminent Parisian physician. I do not know that it is of much importance either with reference to the diagnosis or treatment of pneu. monia. It is interesting as occurring on the side of the inflamed lung; but where it exists there are other more prominent symptoms of the dis. ease, and in some cases of pneumonia it is absent.

EAR-Douche. - Dr. Edw. H. Clarke describes (Boston Med. and Surg. Fourn., May 3oth, 1867) an instrument for cleansing the ear, which, he thinks preferable to the syringe. It consists of a glass jar, of the capacity of about a pint, and of a flexible tube three feet long. One end of the tube is attached to the jar, and the other end is furnished with a nozzle appropriate for the ear. By elevating the jar to a greater or less height, a continued stream of water is poured into the meatus with sufficient force to cleanse it thoroughly, and to do so without pain to the patient. He has used this instrument for the purpose of cleansing the ear in a large number of cases, and is satisfied with its. actions. An instrument, acting on the same principle, has been used for some years for administering injections per rectum. 